\begin{tabular}{|c|c|c|c|c|c|}
\hline MUNIBE Antropologia-Arkeologia & $\mathrm{n}^{\circ} 72$ & $141-156$ & DONOSTIA & 2021 & ISSN 1132-2217 • eISSN 2172-4555 \\
\hline
\end{tabular}

\title{
El revestimiento pintado del Departamento XX de Cabezo Redondo (Villena, Alicante). Caracterización de un elemento constructivo singular
}

\author{
The painted-coating of the XX Department from Cabezo Redondo \\ (Villena, Alicante). Characterization of a singular constructive element
}

PALABRAS CLAVES: Edad del Bronce, Sudeste Península Ibérica, mortero pintado, arquitectura, difracción de Rayos X, Raman. GAKO-HITZAK: Brontze Aroa, Iberiar Penintsulako Hego Ekialdea, mortero margotua, arkitektura, X izpien difrakzioa, Raman. KEY WORDS: Bronze Age, Southeastern Iberian Peninsula, Painted mortar, Architecture, X-Ray Diffraction, Raman.

\section{Alberto DORADO ALEJOS ${ }^{(1)}$, María PASTOR QUILES(2), Gabriel GARCÍA ATIÉNZAR(3), Virginia BARCIELA GONZÁLEZ ${ }^{(4)}$, Mauro S. HERNÁNDEZ PÉREZ(5)}

\section{RESUMEN}

La arquitectura es una de las primeras expresiones de la cultura humana. Su desarrollo ha sido una constante a lo largo de los siglos, especialmente a partir de la aparición de los procesos de sedentarización, momento en el cual se aprecia la combinación de diferentes materiales destinados a mejorar la durabilidad y el aislamiento de las construcciones. El desarrollo de revestimientos de las paredes permitió la aparición de superficies lisas sobre las cuales plasmar los códigos ideológicos de las comunidades humanas o, simplemente, desarrollar sus valores estéticos. En este trabajo se presenta el primer fragmento documentado de revestimiento con restos de pintura procedente del yacimiento de la Edad de Bronce de Cabezo Redondo (Villena, Alicante), sobre el cual se han desarrollado un amplio conjunto de analíticas no destructivas con el objetivo de determinar no solo sus características físicas y de composición, sino también la intencionalidad ornamental de la pintura.

\section{LABURPENA}

Gizakion kulturaren lehen adierazpenetako bat da arkitektura. Mendeetan zehar, etengabe garatzen joan da, batez ere, sedentarizazio-prozesuak agertu zirenetik. Une horretatik aurrera, material ezberdinak nahasten hasi ziren, eraikuntzen iraunkortasuna eta isolamendua hobetzeko. Hormen estaldurak garatzearekin batera, gainazal lauak agertu ziren, giza komunitateen kode ideologikoak islatzeko edo, besterik gabe, balio estetikoak garatzeko aukera ematen zutenak. Lan honetan, Brontze Aroko Cabezo Redondo (Villena, Alicante) aztarnategiko pintura-hondarrak dituen lehen estaldura zati dokumentatua aurkezten da. Azterketa ez-suntsitzaile ugari garatu dira estaldura zati horren gainean, haren ezaugarri fisikoak eta konposizioa zehazteko, baita pinturaren apaindura-helburua zehazteko ere.

\section{ABSTRACT}

Architecture is one of the first expressions of human culture. Its development has been a constant throughout the centuries, especially since the appearance of sedentarization processes, when the combination of different materials to improve the durability and isolation of the constructions is observed. The execution of coverings for walls and other domestic structures made smooth surfaces available, on which to express the ideological codes of human communities or simply to develop their aesthetic values. In this paper, a plastered building fragment with remains of paint from the Cabezo Redondo Bronze Age site (Villena, Alicante) is presented. This is the first decorated fragment of render documented in the settlement. The analysis of the archaeological context in which it appeared allows us to relate it to other similar evidence documented in other Bronze Age sites, inside and outside the area of the El Argar culture. On this fragment a wide set of non-destructive analyses have been developed to preserve its patrimonial value. Firstly, a macroscopic observation with the aim of making a detailed description. Furthermore, mineralogical studies have been carried out to address the composition of the covering and the ornamental band, but also the geological characterization of the deposit environment, to contextualize the origin of the materials used. These analyses have also made possible to explore the manufacturing and maintenance processes of the domestic structures built in Cabezo Redondo, through the study of this singular element from these diverse and complementary approaches.

\footnotetext{
(1) Laboratorio de Arqueometría 'Antonio Arribas Palau', Dpto. de Prehistoria y Arqueología, Universidad de Granada. Campus Universitario de Cartuja CP:18071 Granada (Granada). doradoalejos@ugr.es

(2) Institut Català d'Arqueologia Clàssica. mpastor@icac.cat

(3) Instituto de Investigación en Arqueología y patrimonio Histórico (INAPH)-Área de Prehistoria. Universidad de Alicante. g.garcia@ua.es

(4) Instituto de Investigación en Arqueología y patrimonio Histórico (INAPH)-Área de Prehistoria. Universidad de Alicante. virginia.barciela@ua.es

(5) Instituto de Investigación en Arqueología y patrimonio Histórico (INAPH)-Área de Prehistoria. Universidad de Alicante. mauro.hernandez@ua.es
} 


\section{INTRODUCCIÓN}

El estado actual de la investigación permite plantear que, a lo largo de la Prehistoria reciente de la península ibérica, se utilizaron distintos materiales en las actividades constructivas. Por un lado, recursos naturales como la madera, la piedra y la tierra, para los que se ha planteado una procedencia local (Rivera, 2009; Martínez Mira et al., 2014: 373; entre otros). Por otra parte, también residuos de las actividades económicas cotidianas propias de sociedades agricultoras y ganaderas, como la paja o el estiércol (Ayala et al., 1989: 285; Pastor, 2016: 31, Fig. 7), así como otros elementos de elaboración antrópica, como el cordaje de fibras vegetales. Estos materiales fueron empleados mediante distintas técnicas constructivas, dando lugar a estructuras y espacios cuya variabilidad es amplia a lo largo de la Prehistoria reciente.

Uno de los materiales más utilizados a lo largo de esta etapa fue la tierra, siendo habitual detectar su uso conformando estructuras macizas, como relleno o como elemento de unión de otros materiales con los que se combina. Del mismo modo, se ha registrado su aplicación como recubrimiento, alisando las superficies externas. No obstante, mucho menos común es el tratamiento de estas superficies mediante el añadido de capas diferenciadas de revestimientos, enfoscados y enlucidos que, en algunos casos, fueron decoradas mediante distintas técnicas, como la pintura.

El empleo de revestimientos en las distintas superficies de las edificaciones -desde los alzados a las estructuras de equipamiento o mobiliario domésticorelacionado con la protección frente a distintos agentes erosivos, pero también con fines ornamentales, se habría practicado en los asentamientos prehistóricos peninsulares desde el Neolítico. Así, por ejemplo, se menciona la presencia de enlucidos en los restos constructivos de barro recuperados en el Tossal de les Basses (Alicante) (Rosser y Fuentes, 2007: 17) y en los Castillejos de las Peñas de los Gitanos (Montefrío, Granada), señalándose, para niveles del Neolítico medio, evidencias de "estuco pintado de rojo" (Rubio, 1985: 156; Pellicer, 1995: 97). En cronologías calcolíticas, se indica la presencia de enlucidos en un mayor número de enclaves, como en El Malagón (Cúllar, Granada) (Moreno, 1993: 706) o en el Cerro de la Virgen (Orce, Granada), donde se cita la existencia de restos de pigmento blanco en los recubrimientos (Schüle y Pellicer, 1966: 8). También presentan enlucidos los elementos constructivos de la Illeta dels Banyets (El Campello, Alicante) (Gómez, 2006: 277), La Torreta-El Monastil (Elda, Alicante) (Jover, 2010; Martínez y Vilaplana, 2010; Pastor, 2019: 207, Fig. 97) o Les Moreres (Crevillente, Alicante) (Pastor, 2019: 256, Fig. 137).

Durante la Edad del Bronce, el empleo de capas de revestimiento en las estructuras construidas se documenta de forma habitual en diferentes contextos. Podemos citar los casos de Hoya Quemada (Mora de Rubielos, Teruel) (Burillo y Picazo, 1986: 10), Cerro de
El Cuchillo (Almansa, Albacete) (Hernández Pérez et al., 1994: 53, 61, 194), Orpesa la Vella (Oropesa del Mar, Castellón) (Gusi y Olària, 2014: 28), Lloma de Betxí (Paterna, Valencia) (De Pedro, 1998: 47, 299, Lám. VII. 1), Barranco Tuerto (Villena, Alicante) (Jover y López, 2005: 104) y Terlinques (Jover y López, 2016: 435; Pastor, 2019: 387-389, Figs. 253, 254). Del mismo modo, la práctica del enlucido de superficies se encontraría extendida en los asentamientos argáricos, como se ha constatado en El Rincón de Almendricos (Lorca, Murcia) (Ayala et al., 1989: 282), La Bastida y Tira del Lienzo (Totana, Murcia) (Lull et al., 2015: 76 y 168), La Almoloya (Pliego, Murcia) (Lull et al., 2015: 75) o Laderas del Castillo (Callosa de Segura, Alicante) (Pastor, 2019: 305-307, Figs. 176-179). Los revestimientos pintados se documentan, asimismo, en algunos enclaves del Bronce Final, como Caramoro II (Elche, Alicante) (García Borja et al., 2010: 50-51) y, de manera más habitual, en la Edad del Hierro.

El objetivo de este estudio es presentar un fragmento de revestimiento localizado en el poblado de la Edad del Bronce de Cabezo Redondo (Villena, Alicante) (Fig. 1). La arquitectura doméstica de este asentamiento destaca no solo por sus extraordinarios revestimientos, que cubren tanto alzados como el mobiliario doméstico, sino también por su notable estado de conservación (Hernández et al., 2016) (Fig. 2). Un elemento que difiere de los ejemplos anteriormente citados, pero también de los reconocidos en el propio asentamiento, es que el revestimiento aquí analizado presenta una superficie acabada con una banda pintada. Fue localizado en el interior de un relleno, en posición secundaria, pudiendo tratarse de parte del revestimiento de un alzado o de una estructura inmueble de equipamiento doméstico, tan comunes en este asentamiento. Su análisis cronoestratigráfico y de los diferentes componentes mineralógicos permiten ahondar en la caracterización precisa de unas técnicas constructivas y ornamentales que denotan, una vez más, la extraordinaria singularidad de este enclave del Bronce Tardío.

\section{EL CONTEXTO ARQUEOLÓGICO Y CRONOLÓGICO}

El pequeño fragmento de revestimiento se recuperó del corte 1 planteado durante la campaña de 1998 en el sector central del Departamento XX (Fig. 1), espacio en el cual se habían documentado en años anteriores hasta dos momentos de ocupación superpuestos (Hernández et al., 2016: 48-52). Esta intervención se planteó a modo de sondeo de 2 × 2 m con la intención de concretar la secuencia estratigráfica existente entre el pavimento más antiguo y la base geológica. Concretamente, la pieza procede de la UE 3005, un estrato de textura endurecida y de color marrón que se superpone a la roca base (UE 3008), así como a diferentes estratos (UE 3006 -arcillas verdosas- y UE 3007 -yeso disgregado-). Por encima, se detectaron otras unidades de relleno que se acopiaron en este espacio hasta con- 


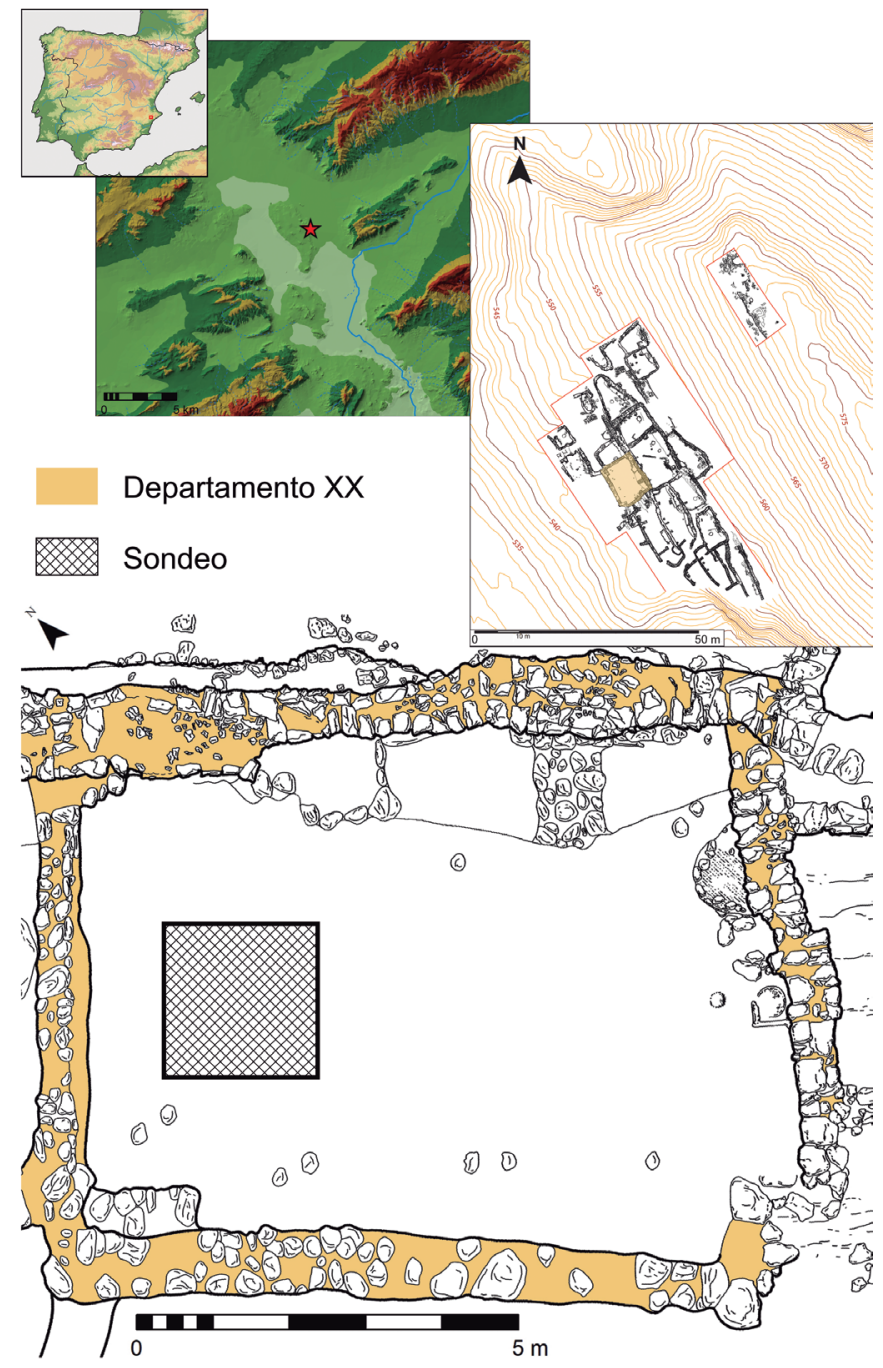

Fig.1. Localización de Cabezo Redondo y planimetría del Departamento XX, con indicación del sondeo en e cual se documentó el fragmento analizado. / Location of Cabezo Redondo and planimetry of Department XX, with indication of the drill hole in which the analyzed fragment was documented.

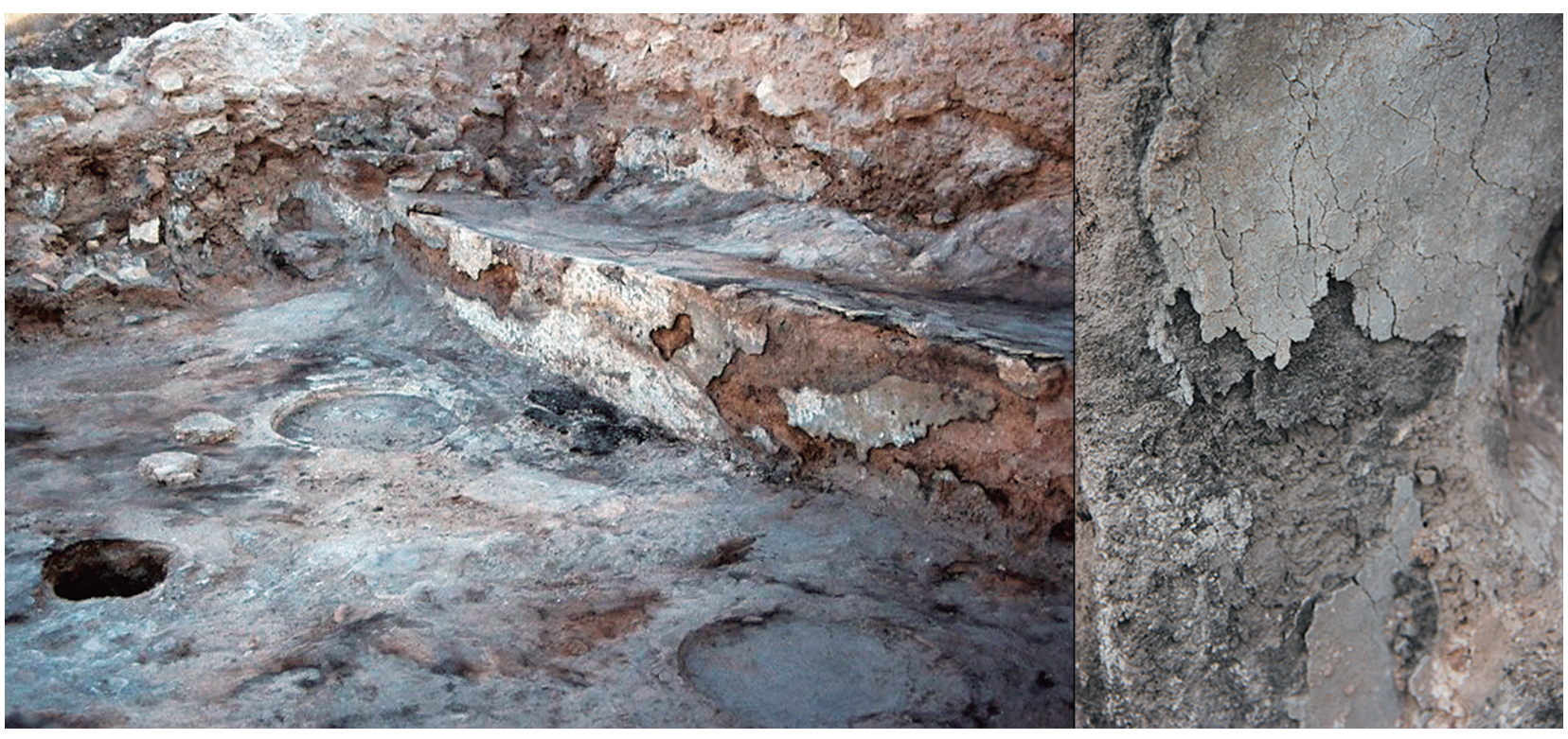

Fig.2. Revestimientos de las estructuras domésticas del Departamento XXV (izquierda) y detalle del revestimiento de una de las paredes del Departamento XXXII (derecha). / Cladding of the domestic structures of Department XXV (left) and detail of the cladding of one of the walls of Department XXXII (right). 
seguir una superficie horizontal sobre la cual colocar el primer pavimento -nivel de uso- del departamento. Como ocurre en otros ambientes del asentamiento, su construcción se inició con el levantamiento de los muros de delimitación que apoyan directamente sobre la base geológica. Con posterioridad, el buzamiento de la roca se superaba a través del acopio de sedimentos y abundantes piedras de pequeño y mediano tamaño, además de basura secundaria ${ }^{1}$ de características muy heterogéneas -fragmentos de cerámica y de fauna, instrumental lítico amortizado, restos constructivos, etc.-, procedentes de diferentes lugares del asentamiento y asociados a ocupaciones previas. Finalmente, sobre estos rellenos se colocaban tanto los pavimentos como las diferentes estructuras domésticas o artesanales.

No se poseen dataciones absolutas directamente asociadas al nivel en el que se recuperó el fragmento analizado, aunque sí de la estratigrafía que conforma la terraza constructiva sobre la cual se asienta el Departamento $X X$, erigido sobre un potente paquete de relleno sobre el cual también se levantaron otras construcciones, como el Departamento XXI o el Espacio Abierto (Tabla 1). Estas dataciones permiten situar la construcción de esta terraza con anterioridad a la horquilla 1665-1514 cal BC, rango definido por la suma de probabilidades de las dos dataciones sobre muestra de vida corta obtenidas para este contexto. Con anterioridad a este momento debió producirse una importante reordenación urbanística del asentamiento, con la más que probable destrucción/desmantelamiento del espacio del cual procedería el revestimiento pintado, así como de algunas de las sepulturas localizadas en los ámbitos domésticos, según testimonian los restos humanos y materiales constructivos dispersos a lo largo de la estratigrafía de estos niveles de relleno. Sobre estos rellenos se levantarían nuevos espacios, algunos de los cuales presentan una larga secuencia de ocupación, como el Departamento XX, que presenta varios episodios de destrucción/reconstrucción.

\section{MÉTODOS Y TÉCNICAS}

El análisis de este fragmento de revestimiento parte de la observación macroscópica de la pieza, así como de una descripción detallada, con el objetivo de definir sus dimensiones y diferentes capas estructurales y decorativas. Esta primera aproximación resulta esencial para abordar otros aspectos, como son la composición del recubrimiento y la banda ornamental, hasta la fecha la única documentada en el yacimiento. Del mismo modo, también resulta esencial realizar una caracterización geológica del entorno de Cabezo Redondo que permita conocer las litologías regionales y contextualizar la procedencia de los materiales locales empleados. Todo ello permitirá obtener información relacionada con los recursos abióticos del entorno y su aprovechamiento, así como con los procesos de manufactura y mantenimiento de las estructuras domésticas, atendiendo a los conocimientos técnicos de estas poblaciones.

Para alcanzar los objetivos planteados en el trabajo se ha procedido, en primer lugar, a la observación y obtención de fotografías de detalle mediante una lupa estereoscópica Leica M80, con oculares de 10x y cámara EZ-350 a la que se han aplicado diferentes aumentos. El software utilizado ha sido el sistema de adquisición de datos Las Interactive Measurement Module V.4 de Leica y las fotografías han sido tratadas, posteriormente, mediante el plug-in DStretch $®$ desarrollado por Gary Hein para el programa ImageJ. Este tratamiento permite detectar la inclusión de determinados materiales en la matriz del mortero. La observación mediante aumentos ha permitido realizar un análisis traceológico de las diferentes superficies, cuyos resultados han sido contrastados con datos obtenidos de diferentes programas experimentales.

Con el fin de definir de forma pormenorizada algunos aspectos, como los elementos compositivos del resto constructivo, la granulometría aproximada de

\begin{tabular}{|c|c|c|c|c|c|}
\hline Referencia & BP & $2 \sigma$ & $\delta 13 C(\%)$ & Contexto & Muestra \\
\hline Beta-189004 & $3350 \pm 70$ & $\begin{array}{c}1876-1842(2,9 \%) \\
1820-1797(1,5 \%) \\
1781-1495(89,6 \%) \\
1478-1457(1,3 \%)\end{array}$ & $-20,3$ & Espacio abierto-Construcción & Humano \\
\hline Beta-361368 & $3310 \pm 30$ & $1661-1509(95,4 \%)$ & $-20,6$ & Espacio abierto-Construcción & Hordeum sp. \\
\hline Beta-181405 & $3180 \pm 70$ & $1617-1282(95,4 \%)$ & $-25,0$ & Dpto. XX-Destrucción fase 1 & Semillas \\
\hline Beta-181404 & $3080 \pm 60$ & $\begin{array}{c}1496-1474(1,8 \%) \\
1462-1192(92,7 \%) \\
1143-1132(0,9 \%)\end{array}$ & $-25,0$ & Dpto. XX-Destrucción fase 2 & Carbón \\
\hline
\end{tabular}

Tabla 1: Dataciones de ${ }^{14} \mathrm{C}$ de los diferentes eventos asociados al Departamento XX de donde procede el fragmento analizado. / $\mathrm{C}^{14}$ data obtained from samples assigned to the first level of occupation of Department XX where the analysed fragment comes from.

\footnotetext{
1 Schiffer (1983) definió la basura secundaria como aquella introducida intencionalmente por los habitantes del poblado, en este caso para aprovecharla como parte de la cimentación y aterrazamiento de los suelos. La materialidad asociada a estos contextos se caracterizará por artefactos erosionados y fracturados, así como ecofactos dispersos y con gran heterogeneidad al derivarse de acciones antrópicas previas y desestructuradas de su contexto original.
} 
los antiplásticos, la zona afectada por la pintura, entre otros, se han realizado una serie de microfotografías de las diversas áreas del fragmento. Para ello se ha empleado un microscópico de luz invertida MEF4 A/M de Leica conectado a una cámara Canon EOS serie 1200D.

La caracterización mineralógica del mortero se ha realizado mediante difracción de Rayos X con un difractómetro Bruker D8 DISCOVER, con detector DECTRIS PILATUS3R 100K-A, microfuente de cobre, haz ajustable y plataforma automática $X Y Z$. Las condiciones de trabajo han sido $\theta-2 \theta, \Delta \theta=0,04^{\circ}$ con $1 \mathrm{~s}$ por paso, $2 \theta=5-70^{\circ}$, con una potencia de trabajo de $40 \mathrm{kV}$ y 40 $\mathrm{mA}$. Por último, los difractogramas resultantes han sido analizados con el programa XPowder (Martín Ramos, 2006) y la base de datos PDF2 del International Centre for Diffraction Data. Se ha utilizado el estándar del cuarzo para corregir las posibles desviaciones (Chisholm, 2005), siendo la ficha Quartz (85-0796) de la base de datos PDF2.

Por último, se ha aplicado Espectroscopía Raman con el objeto de identificar los pigmentos localizados en la superficie del fragmento estudiado. Esta técnica se encuentra ampliamente probada en nuestra disciplina sobre otros materiales como arte rupestre, paramentos de villae romanas o en la caracterización de los pigmentos que ornamentan las cerámicas arqueológicas (Zuo et al., 1999; Colomban et al., 2004; Edwards y Chalmers, 2005; Sendova et al., 2005; Sandalinas et al., 2006; Striova et al., 2006; De Benedetto et al., 2011; Ayora-Cañada et al., 2012).

\section{CARACTERIZACIÓN DEL REVESTIMIENTO}

\subsection{Descripción morfológica y estructural de la pieza}

Se trata de un pequeño fragmento -20,23 x 26,46 $\mathrm{mm}$ - de morfología irregular del revestimiento de un alzado que pudo formar parte de una unidad constructiva mayor, sin que pueda descartarse por completo que se hubiera tratado del enlucido de otro elemento, también susceptible de ser pintado del mismo modo, como pudo ser una estructura inmueble de equipamiento doméstico. Tras un primer análisis visual se pudieron identificar varias capas que conformaban este revestimiento. Del mismo modo, una primera exploración visual permitió detectar restos de pigmento en la superficie de la pieza, conformando una banda de 23,30 mm de longitud -la totalidad de la anchura del fragmento- y 2,5 mm de anchura. Su caracterización precisa se ha llevado a cabo en el análisis macroscópico y de componentes de la pieza.

\subsection{Caracterización geológica del entorno}

El Cabezo Redondo se localiza al NW de la actual provincia de Alicante (España), en la comarca del Alto Vinalopó, uno de los corredores que comunica esta región con la Meseta (Fig. 3). Geológicamente está integrado en la cordillera Bética, caracterizada por plegamientos en sentido ONO-ESE debido a los efectos compresivos de la orogenia Alpina. La existencia en esta región durante el Mesozoico y Cenozoico de dos ámbitos paleogeográficos diferentes dio lugar a que estén representados tanto el sistema Prebético como, mayormente, el Subbético. Asimismo, en las depresiones se desarrollaron cuencas neógeno-cuaternarias en las que, a favor de fracturas y fallas, han ascendido por diapirismo materiales triásicos, proceso que se produjo desde, al menos, el Mioceno hasta la actualidad. Un ejemplo singular de este esquema geológico es la propia falla del Vinalopó, un accidente tectónico -con significación paleogeográfica- que separa ambos sistemas, desarrollándose el Prebético hacia el oeste del Vinalopó y el Subbético al este (Pérez-López, 1991; Estévez et al., 2004; Fernández y Pérez-López, 2004; Martin-Algarra y Vera, 2004; Pérez-Valera, 2005; entre otros).

El propio Cabezo Redondo es, en parte, uno de estos afloramientos de origen halocinético, favorecido por la ascensión de las sales a favor de una fractura asociada a la falla del Vinalopó. De este modo, junto a la ascensión de sales, afloraron yesos, arcillas, margas, dolomías y areniscas con micas. Estos asomos diapíricos constituyeron pequeños afloramientos sobre la cobertera neógena, que acabaron destacando en la llanura debido a los procesos de erosión diferencial.

En Cabezo Redondo se identifican dos unidades litológicas. En la base y tramo medio afloran los ya citados yesos blancos y rojos con ocasionales intercalaciones de dolomías correspondientes al Keuper, concretamente a la Formación Yesos de Ayora o K5, de edad Carniense-Noriense (Ortí, 1974). Sobre estas, y coronando el cerro, se disponen dolomías y calizas tableadas que corresponden, principalmente a la unidad carbonatada inferior de la cobertera tabular de la Meseta sur.

Con el fin de caracterizar los materiales más comunes localizados en el entorno inmediato de Cabezo Redondo, es decir el Triásico en su facies margo-calcárea, se obtuvieron dos muestras. La primera (CRV-SED-001) se tomó al oeste del yacimiento, junto a un afloramiento de dolomías tableadas grises, en el que se incluían yesos y arcillas yesíferas con escasas inclusiones (Fig. 3a). La segunda muestra (CRV-SED-002) fue obtenida de un sustrato geológico similar al anterior, de dolomías tableadas grises, yesos y arcillas yesíferas con escasas inclusiones, pero en el que los yesos presentaban tonos más rojizos (Fig. 3b). La caracterización mineralógica ha permitido identificar que en ambos casos se trata de yesos ( $\mathrm{CaSO} 4 \cdot 2 \mathrm{H} 2 \mathrm{O}$ ) (Fig. 4), de modo que las tonalidades responden a las inclusiones de otros compuestos no detectados mediante la DRX, presentes en muy pequeñas proporciones, y que se agregan al yeso durante su fase formativa. 

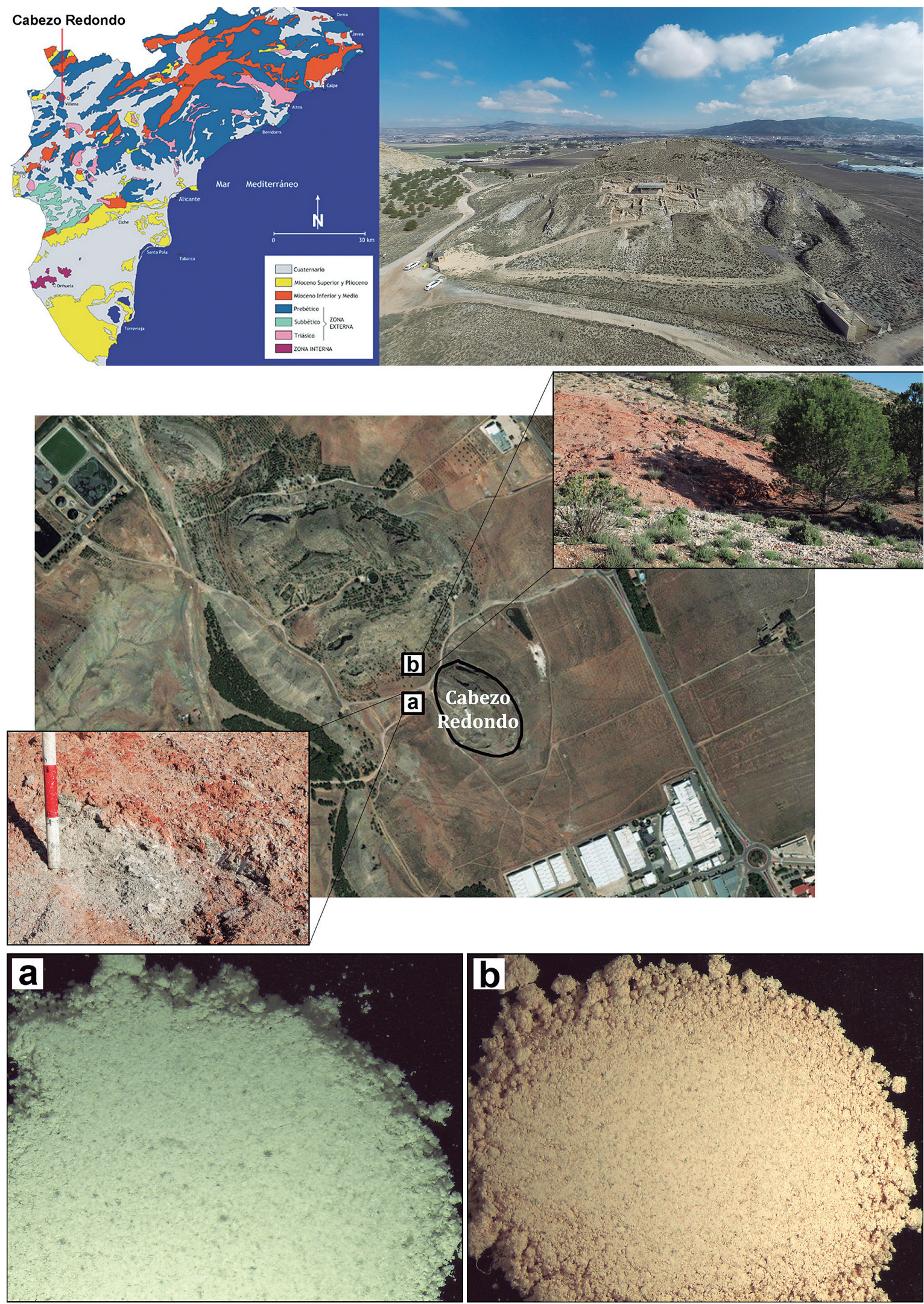

Fig.3. Mapa geológico esquemático de Alicante -IGME- (arriba izquierda); vista aérea de Cabezo Redondo (arriba derecha); lugar de obtención de las muestras de sedimentos CRV-SED-001 (a) y CRV-SED-002 (b). / Geological map of the province of Alicante -IGME- (above left); aerial view of Cabezo Redondo (above right); location of places where the sediment samples CRV-SED-001 (a) and CRV-SED-002 (b) were obtained. 

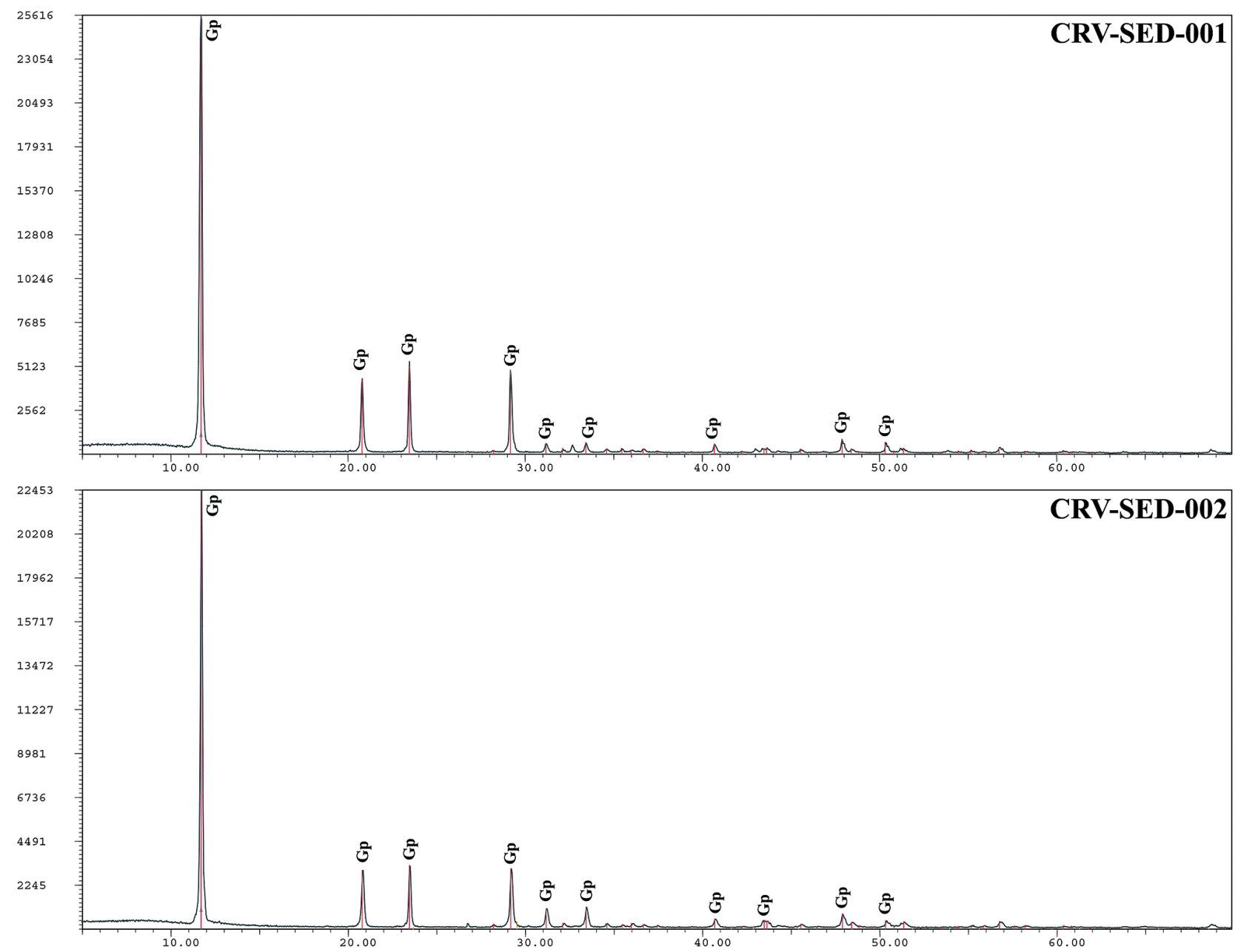

Fig.4. Difractogramas obtenidos de las muestras de sedimentos procedentes del entorno de Cabezo Redondo (Villena, Alicante) que en ambos casos se han definidos como yesos. (Abreviaturas siguiendo a Whitney y Evans, 2010). / Diffractograms obtained from sediment samples from the Cabezo Redondo area (Villena, Alicante) which in many cases have been defined as gypsum. (Abbreviations following Whitney and Evans, 2010).

\subsection{Análisis estructural, componentes y ges- tos técnicos}

Los datos obtenidos a partir de las diversas técnicas aplicadas han permitido realizar una serie de inferencias acerca de los elementos constitutivos del mortero. En este sentido, se han individualizado de forma precisa las capas inicialmente apuntadas gracias a una observación macroscópica que ha permitido identificar las macrotrazas características de los diversos gestos técnicos. Posteriormente, estas capas se han confirmado gracias a un análisis mineralógico.

El estudio macroscópico, realizado a partir del análisis estereoscópico, ha permitido identificar algunas de las trazas generadas en la consecución de este elemento constructivo (Fig. 5a). Se han identificado, así, dos conjuntos de enfoscado/superficie y una banda ornamental pintada.

La superficie externa, a la que se ha denominado Superficie A, presenta en la cara exterior visible unas marcas horizontales, paralelas y próximas entre sí. Este tipo de trazas puede vincularse a instrumentos como espátulas, paletas o llanas de madera cuyo fin sería generar una superficie lisa (Fig. 5b), si bien no puede descartarse el empleo de otros materiales o medios que podrían haber dado lugar a huellas similares. Del mismo modo, estos estigmas pueden responder no solo al instrumento empleado, sino también al arrastre de las partículas del material, pudiendo generarse morfologías de apariencia similar incluso cuando el alisado se realiza con los dedos (Pastor, 2019: 80-81). Finalmente, no puede descartarse que las trazas estén relacionadas con otras estrategias de finalización y pulido de la superficie (Feneulle et al., 2016: 50), aspecto que podría establecerse en futuros trabajos en los que se comparen diferentes tipos de revestimientos.

Uno de los aspectos más relevantes de esta superficie es la identificación de una banda pintada que habría servido de ornamento al elemento original al que perteneció este resto constructivo. El análisis traceoló- 

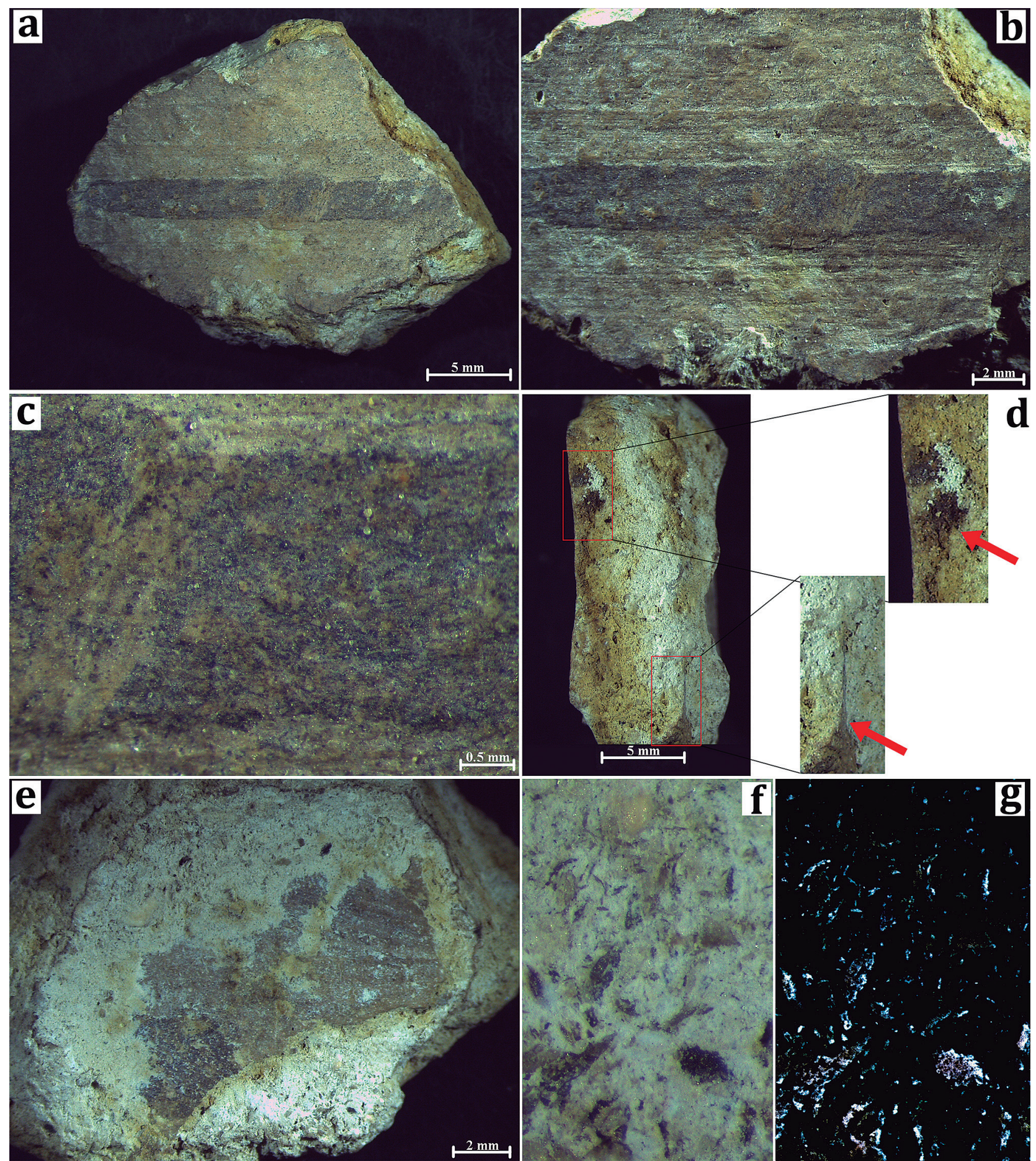

Fig.5. Microfotografías obtenidas de las diversas facetas del revestimiento decorado localizado en el Departamento XX de Cabezo Redondo: a) aspecto general de la muestra vista desde su lado anverso; b) detalles de las macrotrazas identificadas en la superficie que corresponderían a un instrumento; c) detalle del pigmento aplicado sobre la superficie; d) detalles del perfil del elemento en el que puede observarse una segunda capa de revestimiento; e) capa de revestimiento anterior a la superficie vista en la que se observa la aplicación del pigmento; f) detalle de las inclusiones de materia orgánica en la muestra: g) tratamiento de imagen realizado con el programa ImageJ (filtro_labi). / Photomicrographs from the different facets of the decorative coating located in Department XX of Cabezo Redondo: a) general aspect of the sample seen from its obverse side; b) details of the macrotraces identified on the surface that would correspond to an instrument; $c$ ) detail of the pigment applied to the surface; d) details of the element profile in which a second layer of coating can be seen; e) coating layer prior to the surface seen in which the application of the pigment is observed; f) detail of the organic matter inclusions in the sample; $g$ ) image treatment carried out with the ImageJ program (labi_filter) that allows a better observation of the organic inclusions. 
gico revela que fue realizada con una cierta irregularidad en los trazos. La generación de pequeñas rebabas en la zona inferior de la línea parece responder a su aplicación mediante pinceles o herramientas de carácter similar (Fig. 5c)

Atendiendo al análisis macroscópico del perfil de esta primera capa, se observa que la Superficie A no parece diferir sustancialmente del enfoscado realizado para su constitución -Enfoscado A-, por lo que este análisis superficial no permite señalar que se trate de una capa añadida de material más fino y depurado, sino la consecuencia de las distintas pasadas de regularización de la superficie (Fig. 5d-e). La pieza se compone de una segunda capa formada por otro enfoscado y su superficie externa correspondiente -Superficie B y Enfoscado B- que debió realizarse en un momento anterior. La disposición de capas sucesivas de enfoscado o preparación pudo producirse en el marco de un mismo proceso constructivo o, más probablemente, como resultado de la sucesión de actividades de mantenimiento de las estructuras a lo largo del tiempo, hipótesis que cobra fuerza en este caso gracias al resto de analíticas llevadas a cabo (Fig. 6).

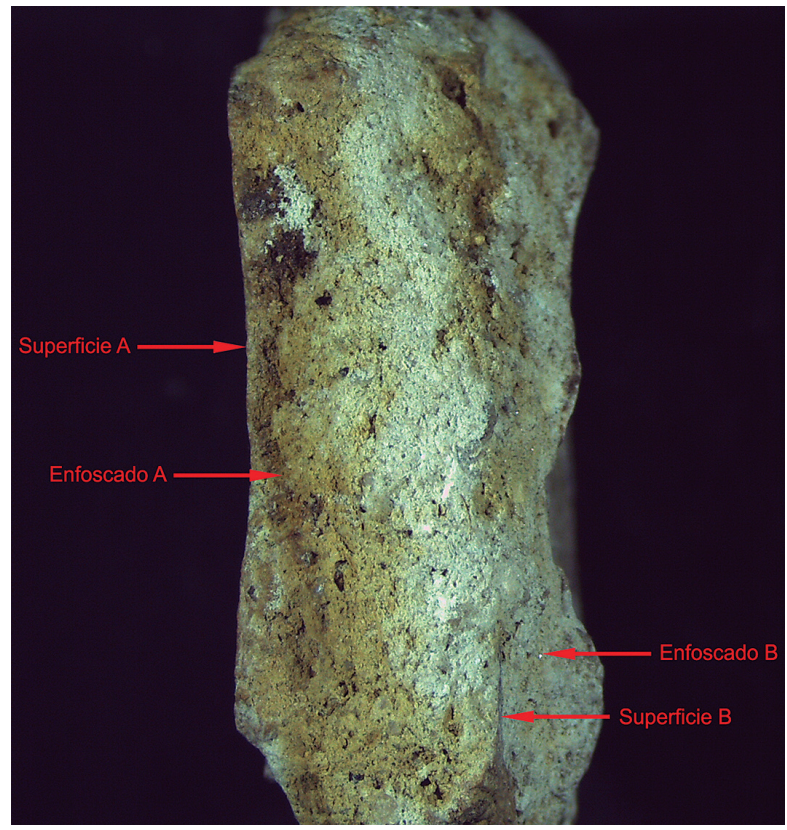

Fig.6. Capas identificadas en el fragmento de estuco de Cabezo Redondo / Layers identified on the Cabezo Redondo plastered fragment.
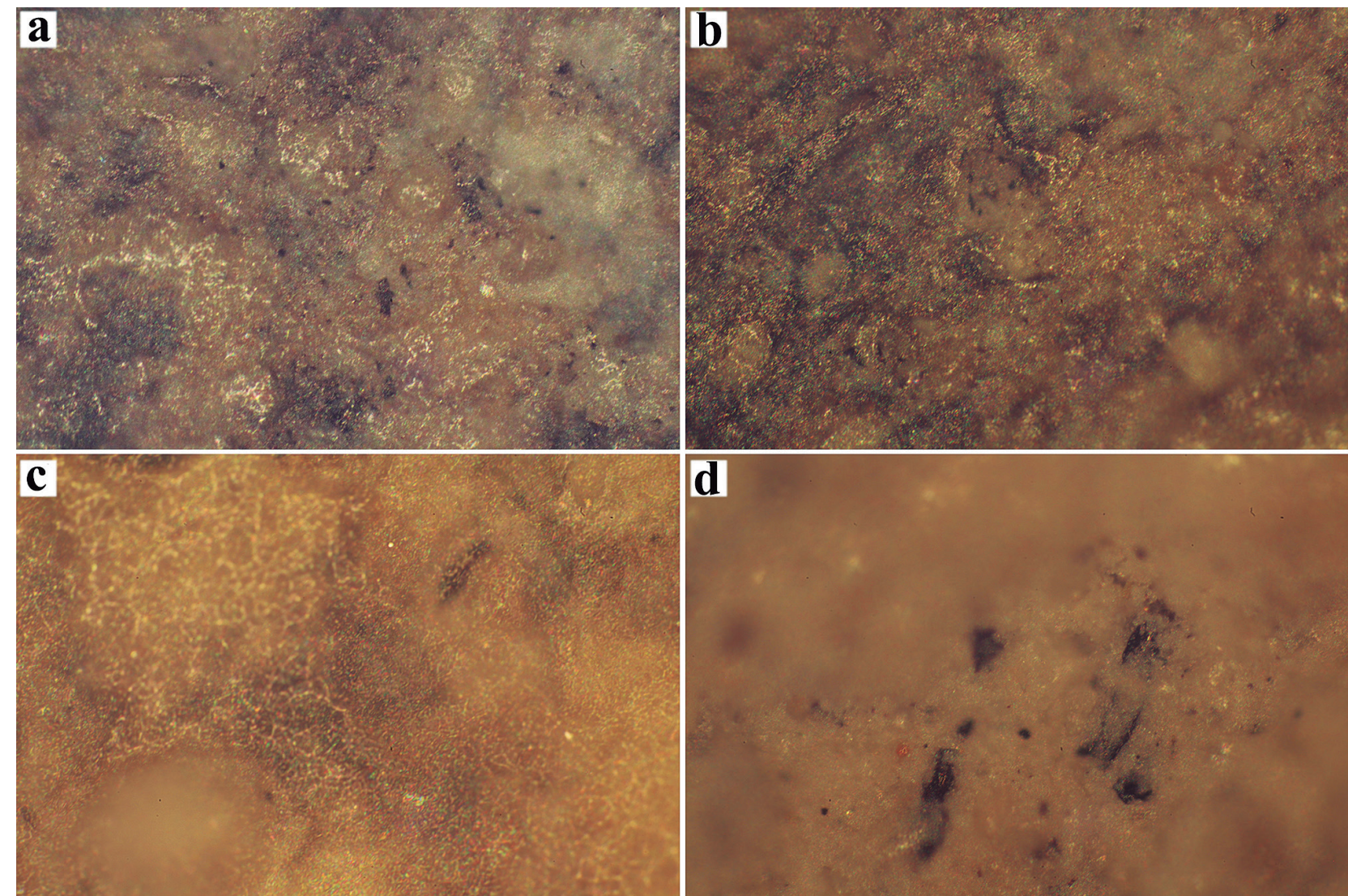

d

Fig.7. Microfotografías obtenidas de las distintas caras del fragmento mediante microscopía de luz reflejada: a) superficie exterior en la que puede observarse la línea de decoración con pequeñas inclusiones orgánicas (20x): b) zonas de contacto entre línea decorativa y base (50x): c) detalle de zona blanca en la que pueden observarse pequeños craquelados (50x), y; d) detalle del reverso de la pieza en la que se incluyen algunos fragmentos de material vegetal (100x). / Microphotographs obtained of the different faces of the fragment by reflected light microscopy: a) outer surface where the decorative line with small organic inclusions can be seen (20x); b) contact areas between decorative line and base (50x); c) detail of white area where small fire cracks can be seen (50x); d) detail of the back of the piece where some fragments of plant material are included (100x). 


\begin{tabular}{|l|c|c|c|c|c|c|c|}
\hline \multicolumn{1}{|c|}{ Localización } & Moscovita & Yeso & Anatasa & Cuarzo & Microclina & Calcita & Dolomita \\
\hline Superficie A & $8^{\prime} 70$ & $14^{\prime} 40$ & $11^{\prime} 70$ & $31^{\prime} 30$ & $11^{\prime} 70$ & $10^{\prime} 90$ & $11^{\prime} 30$ \\
\hline Enfoscado A & 0 & $13^{\prime} 90$ & 13 & 31 & $14^{\prime} 10$ & $13^{\prime} 90$ & $14^{\prime} 10$ \\
\hline Superficie B & 0 & $17^{\prime} 10$ & 0 & $48^{\prime} 10$ & $9^{\prime} 20$ & $24^{\prime} 80$ & 0 \\
\hline Enfoscado B & 0 & $42^{\prime} 60$ & 0 & $9^{\prime} 70$ & 0 & $47^{\prime} 60$ & 0 \\
\hline
\end{tabular}

Tabla 2: Resultados semicuantitativos obtenidos de la lectura de los distintos difractogramas (\% en peso). / Semi-quantitative results obtained from the reading of the different diffractograms expressed in \%.

Señalábamos anteriormente que el estudio macroscópico no revela indicios de que la composición de ambos enfoscados varíe sustancialmente. En cambio, el estudio mineralógico realizado a partir de DRX muestra diferencias en la estructura o recetas diferentes entre ambos momentos e, incluso, entre las capas superficiales que hemos denominado superficies y los morteros que componen los enfoscados. En ambos casos se ha podido identificar restos de materia vegetal que se disponen de forma caótica (Fig. 5f-g). En la caracterización mineralógica realizada mediante difracción de Rayos X se han observado cambios en la composición que debieron tener relación con modificaciones en las recetas de la construcción de morteros (Tabla 2). De este modo, la Superficie A se compone de moscovita, yeso, anatasa, cuarzo, feldespato potásico, calcita y dolomita (Fig. 8a), mientras que el Enfoscado A carece de moscovita, anatasa, feldespato potásico y dolomita, manteniendo las restantes (Fig. 8b). Esta diferencia podría marcar, por tanto, una cierta variación en las materias primas utilizadas para el acabado del revestimiento respecto a la base del mortero. Esta misma diferencia la encontramos en la capa más antigua, de forma que la Superficie B se caracteriza por contener anatasa, cuarzo, feldespato potásico, calcita y dolomita - composición muy similar a la Superficie A- (Fig. 8c), mientras que el Enfoscado B se encuentra carente de anatasa y dolomita (Fig. 8d). Por tanto, los datos parecen evidenciar, por un lado, que no existe una continuidad entre las dos capas que constituyen el revestimiento $y$, por otro, que en ambos casos se utilizan materiales distintos para la superficie y el cuerpo del mortero. A este respecto hemos de señalar, por ejemplo, las mayores cantidades de calcita y yeso en la fase más reciente, mientras que las cantidades de cuarzo -que vinculamos con la aportación de arenas- son mayores en la capa más antigua. Por último, la identificación en la mezcla de dos o más aglomerantes, como son en nuestro caso la calcita y el yeso, nos llevan a definir el mortero como de tipo 'bastardo'.

En cuanto a los componentes que hemos podido identificar como parte de la muestra, en ambas capas se han identificado pequeñas cantidades de cuarzo submilimétrico, bien ordenado, sin una orientación preferencial y con una alta esfericidad. A estos desgrasantes inorgánicos se une la presencia de pequeños restos de materia vegetal como aditivos que se encuentran dispersos en una matriz rica en $\mathrm{CaO}$ y cuya especie no ha podido ser identificada por el momento (Fig. 7d).
La observación realizada mediante Microscopio Electrónico de Barrido también indica una clara matriz calcárea (Fig. 9a-c) en la que pueden observarse pequeñas agregaciones del material como consecuencia de su aplicación, lo que provoca la generación de microporos estriados y alongados. Estos poros son mayores en las zonas de unión entre las diversas capas (Fig. 9b), es decir, entre las respectivas superficies y enfoscados. Con todo, se observan los compuestos típicos de silicatos de cálcico hidratados (CSH y CAH) (Surendran et al., 2017) e, incluso, puede observarse la generación de algunos cristales romboédricos perfectos de calcita dispuestos sobre el calcio amorfo y algunos yesos (Fig. 9d), coexistencia que mejora el proceso de hidratación y, consecuentemente, las propiedades hidráulicas y su resistencia mecánica.

La microscopía de luz reflejada ha proporcionado datos interesantes en cuanto a la aplicación de la pintura en la Superficie A, apuntando a que ésta pudo realizarse mediante un material o instrumento similar a un pincel. Este gesto provocaría cambios en el trazo del pigmento y, con ello, la existencia de unas zonas con mayor densidad y de otras en las que el soporte quedaría al descubierto según se aplicaran sucesivamente las pinceladas (Fig. 7 a-b). La naturaleza del pigmento utilizado en la realización de la banda decorativa ha podido definirse mediante espectroscopia Raman (Fig. 10), cuyo resultado muestra el espectro típico del carbón fósil con sus característicos picos de grafito 1589 $\mathrm{cm}^{-1}$ (conocido como G) (Tuinstra y Koenig 1970) y el pico $1389 \mathrm{~cm}^{-1}$ (conocido como pico D, por disorder) (Cohen-Ofri et al., 2006).

\section{DISCUSIÓN}

Los distintos datos obtenidos en el presente estudio permiten la caracterización de un elemento constructivo de extraordinario interés del yacimiento alicantino de Cabezo Redondo. Sin embargo, no estamos ante una tradición innovadora ya que, como se ha apuntado al inicio, se han identificado enlucidos pintados en distintos asentamientos de la Prehistoria reciente peninsular, pudiendo destacarse los argáricos. Este es el caso de La Almoloya (Pliego, Murcia), donde han sido localizados varios enlucidos en los que pueden observarse representaciones figurativas, triangulares, motivos lineales o lunares que han sido realizados mediante pintura roja (Lull et al., 2015: 100-101) y no a partir de carbón, como es nuestro caso. Otros motivos pintados 

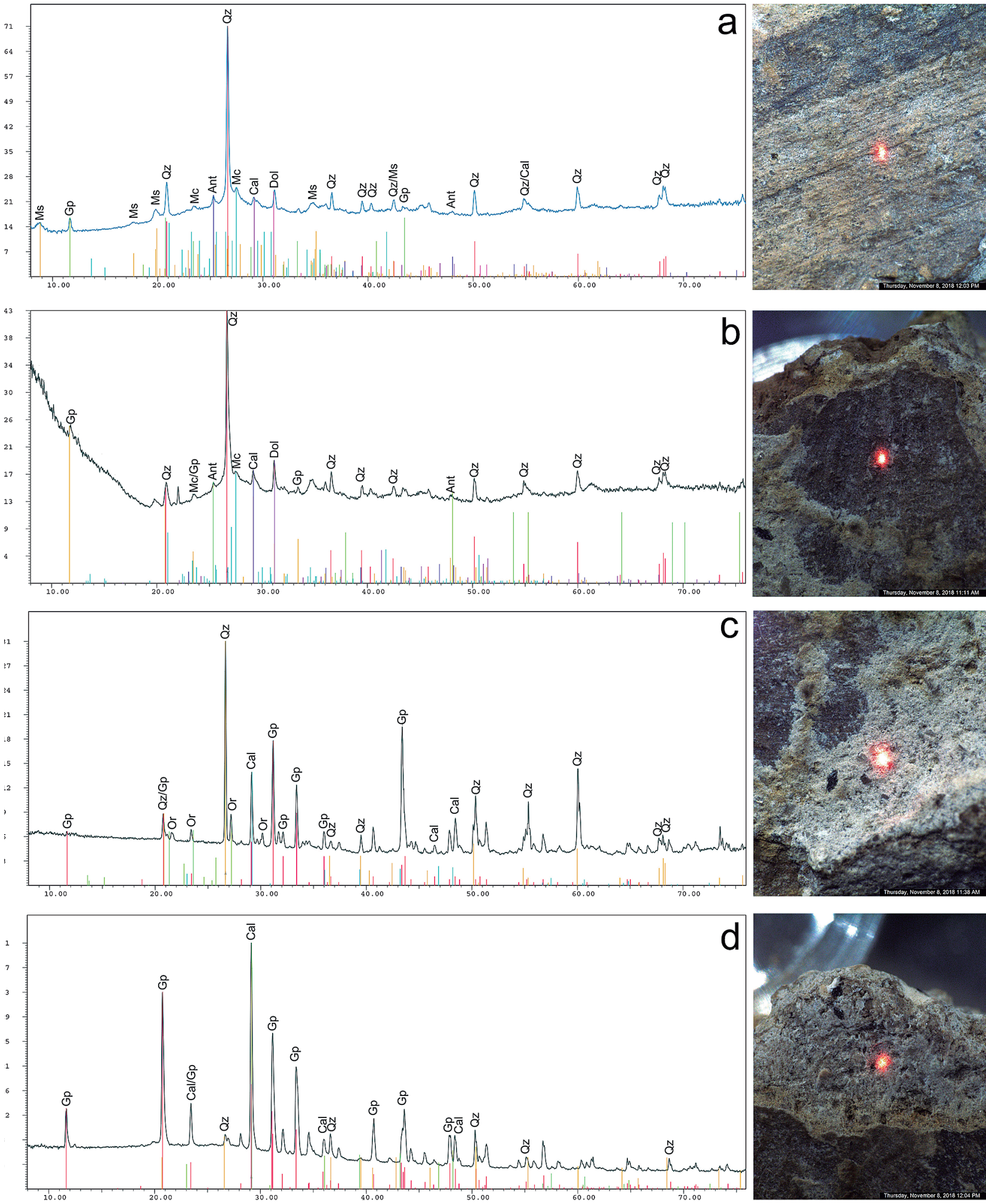

Fig.8. Resultados obtenidos mediante Difracción de Rayos X orientados a la identificación mineralógica de las diversas capas identificadas mediante estereoscopía: a) superficie de la capa más reciente en la que se encuentra la pintura; b) superficie de la capa más antigua; c) mortero de la capa más antigua; d) mortero de la capa más reciente y en la que se encuentra la pintura. (Abreviaturas siguiendo a Whitney y Evans, 2010). / Results obtained by X-Ray Diffraction oriented to the mineralogical identification of the different layers identified by stereoscopy: a) surface of the most recent layer in which the painting is found; b) surface of the oldest layer; c) mortar of the oldest layer; d) mortar of the most recent layer and in which the painting is found. (Abbreviations following Whitney and Evans, 2010). 

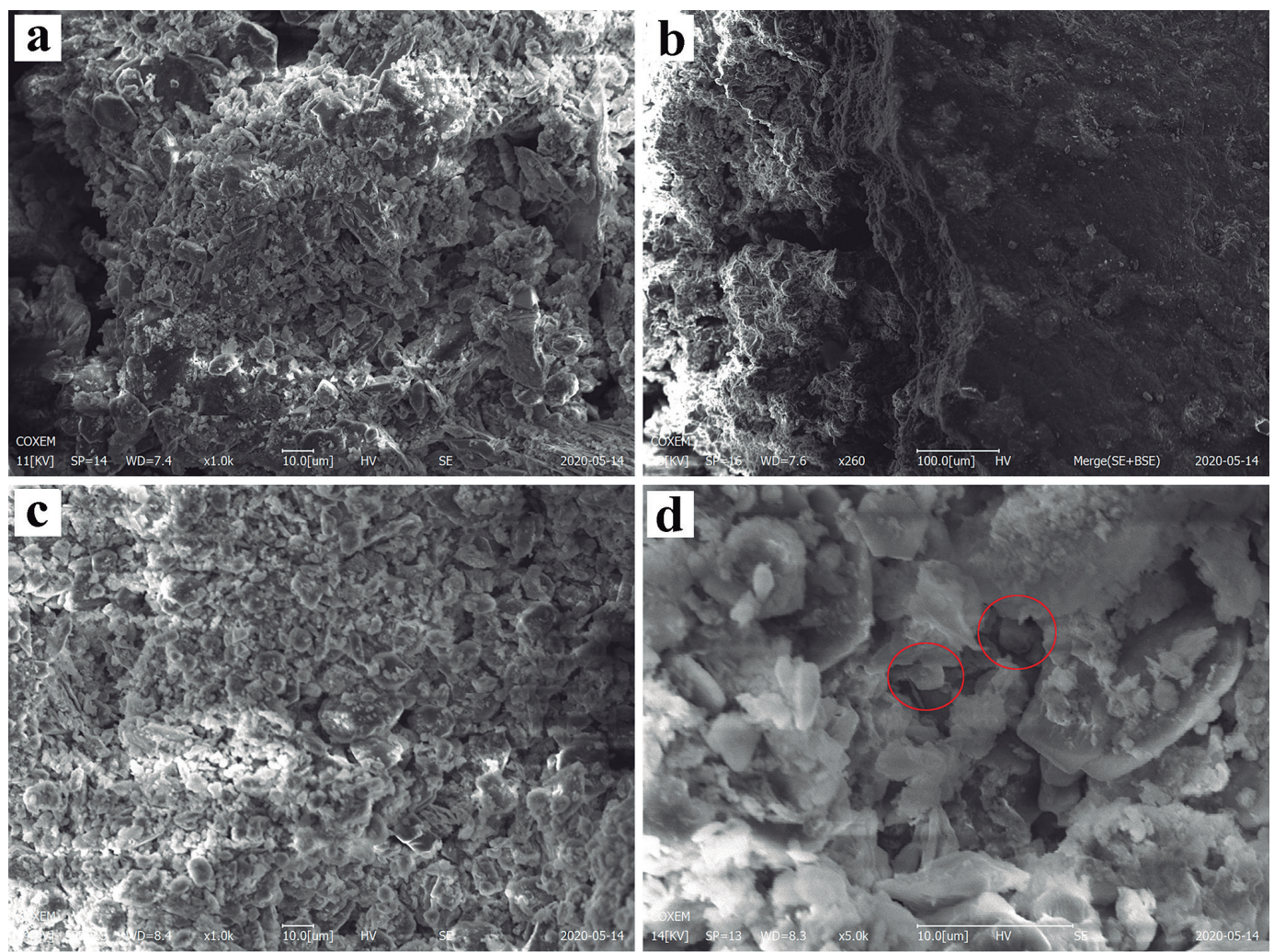

Fig.9. Microfotografías del enfoscado A (a) y su contacto con la Superficie $A(b)$, en la que se encuentra la decoración, y enfoscado más antiguo (c) con detalle de los cristales de carbonato cálcico amorfo y algunos cristales bien formados en rojo (d). / Microphotographs of rendering $\mathrm{A}(\mathrm{a})$ and its contact with Surface $A(b)$, in which the decoration is found, and older rendering (c) with detail of the amorphous calcium carbonate crystals with calcite crystal formations (d).
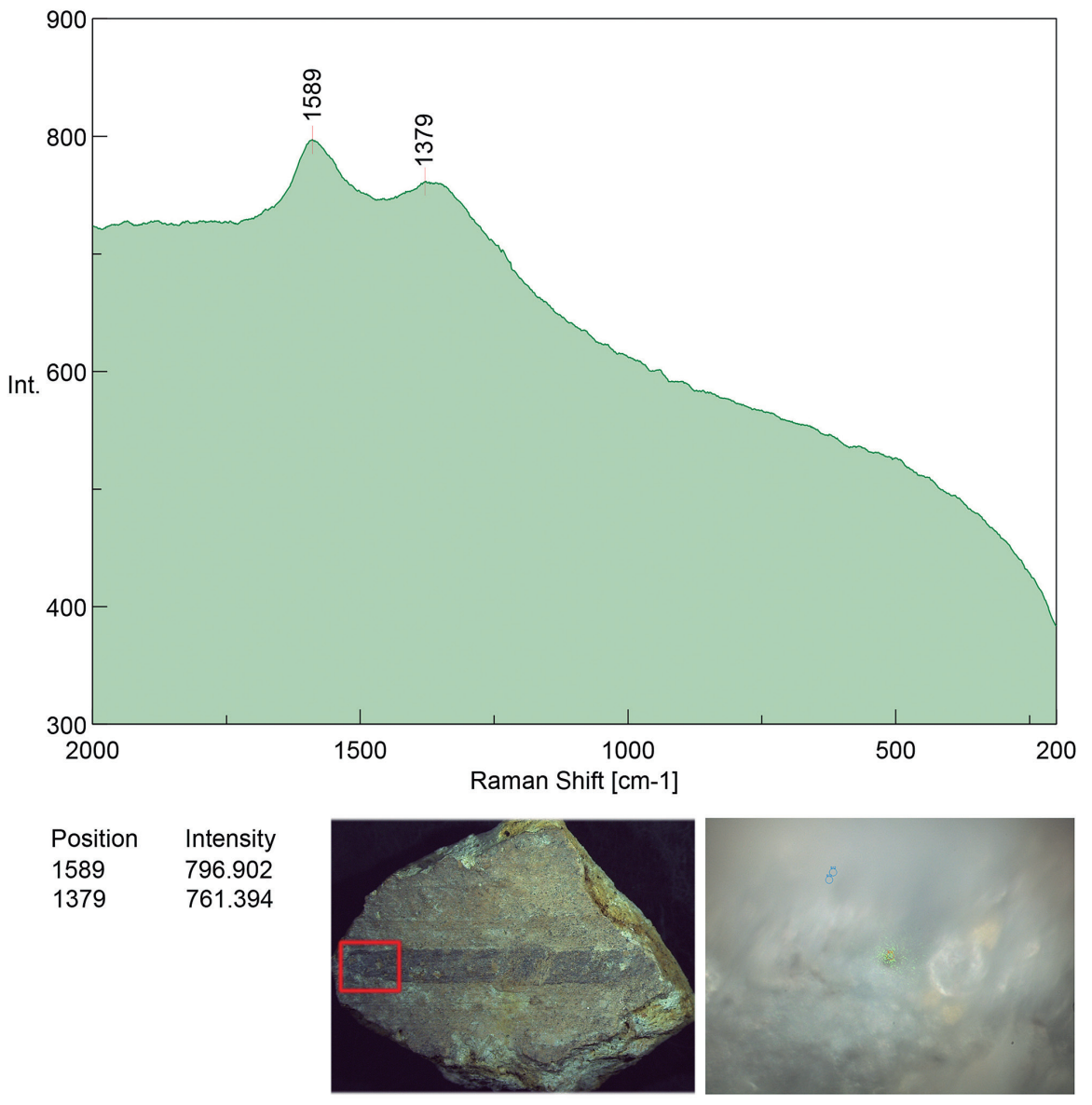

Fig.10. Espectro Raman de la banda decorativa localizada en la Superficie A. / Raman spectrum of the decorative band located on Surface A. 
en restos constructivos de asentamientos argáricos, igualmente con pigmento rojo, han sido apuntados en Cabezo Gordo o de la Cruz (Totana, Murcia) (Ayala, 1986: 332; 2001: 154) y en Laderas del Castillo (Callosa de Segura, Alicante) (Pastor, 2019: 310-314). Fuera del ámbito de El Argar, contamos con el hallazgo de revestimientos pintados con franjas rojas en Orpesa la Vella (Oropesa del Mar, Castellón) (Gusi y Olària, 2014: 65, 74-75).

Aunque en los momentos finales de la Edad del Bronce la práctica del enlucido de los paramentos de las estructuras se mantendrá, no lo hace así la presencia de elementos ornamentales. Sin embargo, pueden citarse algunas excepciones en sitios del Bronce Final, como Cerro de los Infantes (Pinos Puente, Granada), Cerro de Cabezuelos (Úbeda, Jaén), Cerro de la Encina (Monachil, Granada) (Dorado et al., 2015) o Llanete de los Moros (Montoro, Córdoba) (Martín de la Cruz, 1987).

A pesar de las reducidas dimensiones del fragmento conservado y de la aplicación de técnicas no invasivas, se han obtenido datos que nos permiten esbozar, de forma general, los pasos seguidos en la elaboración de este tipo de revestimientos, desde la captación de las materias primas hasta su aplicación en los paramentos y el instrumental empleado.

En relación con el primer paso, la captación de las materias primas, éstas se obtendrían en el entorno próximo del asentamiento, rico en yesos y carbonato cálcico, para proceder a su transformación a partir de la aportación de agua y conseguir su consiguiente apagado. A estos materiales se añadirían arenas -en las que se incluyeron microcristales de cuarzo-, silicatos de cálcico hidratados y elementos vegetales que permiten retrasar la formación de ettringita $\left(\mathrm{Ca}_{6} \mathrm{Al}_{2}\left(\mathrm{SO}_{4}\right)_{3}(\mathrm{O}\right.$ $\mathrm{H})_{12} \cdot 26 \mathrm{H}_{2} \mathrm{O}$ ) (Scrivener y Taylor 1993), un sulfoaluminato de calcio hidratado que puede provocar la formación de grietas, común en morteros ricos en yeso, cementos, etc. Además, estos silicatos de calcio hidratados y elementos vegetales mejoran la formación de CSH y CAH (Surendran et al., 2017), a la vez que su adición permite un mejor amasado de las materias primas.

En cuanto a su aplicación sobre los paramentos, la identificación de macrotrazas en la Superficie A podría responder al uso de herramientas tales como llanas, paletas o espátulas, artefactos todos ellos realizados sobre materia vegetal y que, por su naturaleza orgánica, no se han identificado en el registro arqueológico del sitio. Sin embargo, el tratamiento de la superficie podría no concluir con el alisado, o así parecen indicarlo algunos de los rasgos que podrían vincularse con la aplicación de fuego, el cual tendría como finalidad endurecer la capa más superficial aplicada al mortero (Superficies A y B). La aplicación de fuego podría suponer no sólo la generación de pequeños craquelados en la superficie, documentados mediante microscopía de luz reflejada, sino también el oscurecimiento de la fina capa de sendas superficies y la presencia de un ahumado más o menos regular en las mismas. Ya se ha señalado la posibilidad de que estas termoalteraciones también puedan deberse al incendio asociado a la destrucción del departamento de procedencia del revestimiento, si bien las referencias en otros trabajos a la aplicación intencional de fuego como parte del proceso constructivo para endurecer materiales (Miret, 1992: 69; Shaffer, 1993: 62) permiten considerar esta posibilidad, que deberá ser contrastada en futuros estudios.

El revestimiento muestra dos capas formadas por una superficie alisada y un enfoscado cada una, previamente interpretadas como dos fases diferentes de preparación y mantenimiento de los departamentos de Cabezo Redondo. En este sentido, las analíticas realizadas permiten confirmar esta hipótesis, habiéndose identificado recetas diferentes para las dos capas y entre los enfoscados y superficies de cada una de ellas. Así, la fase más antigua se caracteriza por contener un enfoscado (B) en el que se mezclan arenas con cuarzo, yeso y $\mathrm{CaCO}_{3}$ y una superficie (B) regularizada y alisada en la que las arenas y el yeso se presentan en mayores cantidades. Por su parte, la capa más reciente, en la que encontramos la banda pintada, se caracteriza por tener un enfoscado $(A)$ con menores cantidades de yeso y $\mathrm{CaCO}_{3}$ y menores cantidades de cuarzo, con presencia de dolomita. Estas composiciones resultan similares a las identificadas en otros sitios cercanos como Cabezo Pardo (San Isidro/Granja de Rocamora, Alicante) (Martínez et al., 2014; Jover et al., 2016).

Estos resultados deben interpretarse en dos sentidos. En primer lugar, en relación con una continuidad en el acceso a unas materias primas que, en base a la caracterización geológica, podemos asociar al entorno próximo del sitio, asentado sobre dos unidades litológicas en las que se registran dolomías tableadas grises jurásicas y yesos del Triásico (IGME, Hoja 845: Yecla). En segundo lugar, a lo que podría ser una mejora de la receta empleada y, por tanto, a cambios en el conocimiento de la preparación de morteros entre los dos momentos detectados. Sin embargo, por el momento, no se han podido identificar claramente los procesos de transformación de las materias primas empleadas en los elementos constructivos, pudiendo corresponder los restos vegetales identificados en los enfoscados, probablemente, a estabilizantes de la mezcla (Vilaplana et al., 2011: 276; Jover et al., 2016: 9), además de servir a modo de aglomerante para obtener una mayor cohesión a la vez que se aligera peso.

A modo de finalización del revestimiento, se aplicó una banda ornamental en la Superficie A. Se habría realizado a partir de materia vegetal carbonizada, según se deriva de los resultados obtenidos mediante espectroscopía Raman. Esta banda pudo haber sido ejecutada con un pincel, como se observa a partir del estudio estereoscópico y microscópico con luz refle- 
jada, dato que relacionamos con la identificación de macrotrazas que permiten plantear el uso de ciertos artefactos que, nuevamente, por sus características, no dejan apenas presencia en el registro arqueológico. El empleo de estas herramientas ha sido constatado en otros enclaves como Las Pilas-Huerta Seca (Mojácar, Almería) y Terrera Ventura (Tabernas, Almería) en momentos anteriores para la elaboración de la decoración cerámica pintada (Dorado, 2018: 11, Fig. 2), técnica que habría perdurado en el tiempo. Sin embargo, no descartamos otras opciones como el uso de carbón a modo de pigmento negro.

\section{CONCLUSIONES}

Los resultados obtenidos en el presente estudio han permitido caracterizar un elemento constructivo singular localizado en Cabezo Redondo, un enclave cuyo primer estudio geomorfológico y sedimentológico ya reveló que los habitantes del poblado debieron tener un amplio conocimiento de las propiedades de los materiales geológicos del entorno (Fumanal et al., 1996).

Siguiendo las propuestas de estudio iniciadas por diferentes autores para este tipo de restos constructivos (Feneuille et al., 2016; Jover et al., 2016; Pastor, 2017) y continuando con metodologías seguidas por otros (Melis y Albero, 2017; Moreno y Compaña, 2018), se ha podido definir para este fragmento de revestimiento su estructura en capas, así como la caracterización mineralógica de éstas. Por otra parte, también se han determinado los gestos técnicos seguidos para su realización, además de la determinación de la composición de la banda ornamental.

Destaca la particularidad de esta producción que, hasta la fecha, no encuentra paralelos ni en el propio asentamiento de Cabezo Redondo ni en ningún otro enclave del Sudeste peninsular, en los que los distintos motivos se realizan a partir de pigmentos de tonos rojizos, muy probablemente óxidos de hierro. Por el contrario, en Cabezo Redondo, donde es muy común el uso de revestimientos ante la necesidad de aislar los zócalos y, sobre todo, el recrecimiento de las propias alzadas de las estructuras, la aplicación de las distintas técnicas de análisis presentadas han hecho posible observar las que posiblemente fueron las actividades de mantenimiento, sin que por el momento podamos definir la periodicidad de las mismas, es decir, el lapso temporal que existe entre las dos fases detectadas en el revestimiento. Estas actividades de mantenimiento también se han documentado en otros sitios de la Prehistoria reciente (Sánchez Romero, 2015), y su uso en la zona puede retrotraerse, al menos, desde momentos neolíticos (Jover et al., 2016) hasta alcanzar las épocas moderna y contemporánea, momentos en los que se incrementa sustancialmente la explotación de los yesos, como evidencia la construcción de distintos hornos vinculados a diversas áreas de extracción tanto en Villena como en el propio Cabezo Redondo (Rizo et al., 2000).

\section{AGRADECIMIENTOS}

El presente estudio ha sido realizado en el marco de la Unidad de Excelencia Archaeometrical Studies. Inside the artefacts \& ecofacts, financiada por el Plan Propio de Investigación y Transferencia de la Universidad de Granada, y los proyectos Mejora de la investigación y externalización del Laboratorio de Arqueometría 'Antonio Arribas Palau' Dpto. de Prehistoria y Arqueología, Universidad de Granada (EQC2018-004880-P) y Origen y conformación del Bronce Valenciano (PID2020115956GB-I00) financiados por el Ministerio de Ciencia, Innovación y Universidades.

\section{BIBLIOGRAFÍA}

Ayala Juan, M.M., 1986. La cultura de El Argar en Murcia. Datos actuales. Un avance para su estudio. In: Homenaje a Luis Siret (1934-1984), 329-340. Junta de Andalucía, Sevilla.

Ayala Juan, M. M., 2001. La Edad de Bronce en la región de Murcia. In: Hernández Pérez, M. S. (Ed.), Y acumularon tesoros. Mil años de historia en nuestras tierras, 151-161. CAM, Alicante

Ayala, M. M., Rivera, D., Obón, C., 1989. Improntas vegetales de adobes procedentes de la casa A del yacimiento argárico en llanura de Rincón de Almendricos, Lorca, Murcia. In: Crónica del XIX Congreso Arqueológico Nacional (Castellón, 1987) 1, 279-291. Universidad de Zaragoza, Zaragoza.

Ayora-Cañada, M. J., Domínguez-Arranz, A., Domínguez-Vidal, A., 2012. Raman Microspectroscopic study of Iberian pottery from the La Vispesa archaeological site, Spain. Journal of Raman Spectroscopy 43(2), 317-322.

Brysbaert, A., 2008. The power of technology in the Bronze Age Eastern Mediterranean. The case of the painted plaster. In: Monographs in Mediterranean Archaeology 12. Equinox, London.

Burillo, F., Picazo, J. V., 1986. El poblado del Bronce Medio de la Hoya Quemada (Mora de Rubielos, Teruel). Seminario de Arqueología y Etnología Turolense. Colegio Universitario de Teruel, Teruel.

Chisholm, J., 2005. Comparison of Quartz Standards for X-ray Diffraction Analysis: HSE A9950 (Sikron F600) and NIST SRM 1878. The Annals of Occupational Hygiene 49(4), 351-358.

Cohen-Ofri, I., Weiner, L., Boaretto, L., Mintz, G., Weiner, S., 2006. Modern and fossil charcoal: aspects of structure and diagenesis. Journal of Archaeological Science 33, 428-439.

Colomban, P., Milande, V., Le Bihan, L., 2004. On-site Raman analysis of Iznik pottery glazes and pigments. Journal of Raman Spectroscopy 35(7), 527-535.

De Benedetto, G. E., Nicolì, S., Pennetta, A., Rizzo, D., Sabbatini, L., Mangone, A., 2011. An integrated spectroscopic approach to investigate pigments and engobes on pre-Roman pottery. Journal of Raman Spectroscopy 42(6), 1317-1323.

De Pedro Michó, M. J., 1998. La Lloma de Betxí (Paterna, Valencia). Un poblado de la Edad del Bronce. Serie de Trabajos Varios del SIP, 94. Diputación de Valencia, Valencia.

Dorado Alejos, A., 2018. El análisis de imagen como aportación metodológica al estudio de las cerámicas pintadas de la Prehistoria reciente: casos de estudio desde el sudeste de la península ibérica. Arqueología Iberoamericana S2, 9-14. 
Dorado, A., Molina, F., Contreras, F., Nájera, T., Carrión, F., Sáez, L. De La Torre, F., Gámiz, J., 2015. El Cerro de Cabezuelos (Jódar, Jaén): un asentamiento del Bronce Final en el Alto Guadalquivir. Cuadernos de Prehistoria y Arqueología de la Universidad de Granada 25, 257-347.

Edwards, H. G. M., Chalmers, J. M., 2005. Raman Spectroscopy in Archaeology and Art History. The Royal Society of Chemistry, Northampton.

Eiland, M. L., Williams, Q., 2000. Infra-red Spectroscopy of ceramics from Tell Brak, Syria. Journal of Archaeological Science 27, 993-1006.

Estévez, A., Vera, J.A., Alfaro, P., Andreu, J.M., Tent-Manclus, J.E., Yébenes, A., 2004. Alicante en la Cordillera Bética. In: Alfaro, P., Andreu, J.M., Estévez, A., Tent-Manclus, J.E., Yébenes, A. (Eds.), Geología de Alicante. Libro guía del XIII Simposio sobre enseñanza de la geología, 39-50. Alicante.

Feneuille, S., Letourneux, J.-P., Bouchar, M., 2016. Protocoles d'étude des mortiers anciens à l'usage des archéologues. Mergoil, Autun

Fernández, J., Pérez-López, A., 2004. Evolución sedimentaria y paleogeográfica del Prebético: Triásico. In: Vera, J.A. (Ed.) Geología de España, 365-366. Sociedad Geológica de España e IGME, Madrid.

Fumanal, M.P., Hernández, M.S., Ferrer, C., Serna, A., Batlle, J., Martínez, J., Bordas, V., 1996. Estudio geoarqueológico de Cabezo Redondo (Villena, Alicante): un yacimiento de la edad de bronce y sus condicionantes medioambientales. Cuaternario y Geomorfología 10(3-4), 5-20.

García, P., Carrión, Y., Collado, I., Montero, I., Muñoz, M., Pérez, G., Roldán, C., Román, D., Tormo, C., Verdasco, C., Vives, J., 2010. Campaña de excavación arqueológica de urgencia en Caramoro II (Elx, Alacant). MARQ. Arqueología y museos 4, 37-66.

Gómez Puche, M., 2006. Estudio de los fragmentos de barro cocido en el yacimiento de la 'Illeta dels Banyets' (El Campello, Alicante). In: Soler Díaz, J.A., La ocupación prehistórica de la Illeta dels Banyets (El Campello, Alicante), 271-280. MARQ, Alicante.

Gusi, F., Olària, C., 2014. Un asentamiento fortificado del Bronce Medio y Bronce Final en el litoral mediterráneo: Orpesa La Vella (Orpesa Del Mar, Castellón, España), Monografies de Prehistòria i Arqueologia castellonenques 10, Servei d' Investigacions Arqueològiques i Prehistòriques, Castellón.

Hernández, M. S., Simón, J.L., López, J. A., 1994. Agua y poder. El Cerro de El Cuchillo (Almansa, Albacete). Excavaciones 1986/1990. Servicio de Publicaciones de la Junta de Comunidades de Castilla-La Mancha, Toledo.

Hernández, M.S., García, G., Barciela, V., 2016. Cabezo Redondo (Villena, Alicante). Universidad de Alicante, Alicante.

Jover Maestre, F.J., 2010. Los materiales constructivos de una pequeña comunidad agropecuaria. In: Jover Maestre, F.J. (Coord.), La Torreta-El Monastil (Elda, Alicante): del IV al III milenio a. C en la cuenca del Vinalopó. Series Excavaciones Arqueológicas X, 111-118. MARQ, Alicante.

Jover, F. J., López, J. A., 2005. Barranco Tuerto y el proceso histórico en el corredor del Vinalopó durante el II milenio BC. Vestigium 1. Monografías del Museo Arqueológico de Villena, Villena.

Jover, F. J., López, J. A., 2016. Nuevas bases para el estudio de las comunidades campesinas de la Edad del Bronce en el Levante peninsular: el asentamiento de Terlinques (Villena,
Alicante). In: Del neolític a l'edat del bronze en el Mediterrani occidental. Estudis en homenatge a Bernat Martí Oliver. Serie de Trabajos Varios del SIP 119, 427-449. Diputación de Valencia, Valencia

Jover, F. J., Pastor, M., Martínez, I., Vilaplana, E., 2016. El uso de la cal en la construcción durante la Prehistoria reciente: nuevas aportaciones para el levante de la península Ibérica. Arqueología de la Arquitectura 13, 1-18

Lull, V., Micó, R., Rihuete, C., Risch, R., Celdrán, E., Fregeiro, M. I., Oliart, C., Velasco, C., 2015. La Almoloya (Pliego, Murcia). Ruta Argárica, Guías Arqueológicas 2, Integral. Sociedad para el Desarrollo Rural-ASOME-UAB, Murcia.

Martín de la Cruz, J. C., 1987. Llanete de los Moros, Montoro, Córdoba. Excavaciones Arqueológicas en España 151. Ministerio de Educación, Madrid.

Martín Ramos, J. D., 2006. XPowder. Programa para análisis cualitativo y cuantitativo por Difracción de Rayos X. MACLA 4-5, 35-44.

Martín-Algarra A., Vera, J.A., 2004. La Cordillera Bética y las Baleares en el contexto del Mediterráneo Occidental. In: Vera, J.A. (Ed.), Geología de España. Sociedad Geológica de España e IGME. Madrid, 352-354.

Martínez, I., Vilaplana, E., 2010. Dos fragmentos constructivos procedentes del yacimiento de la Torreta-El Monastil (Elda, Alicante): análisis mediante diferentes técnicas instrumentales (FRX, DRX, FTIR-IR, TG-ATD, SEM-EDX). In: Jover Maestre, F. J. (Coord.), La Torreta-El Monastil (Elda, Alicante): del IV al III milenio a. C en la cuenca del Vinalopó. Series Excavaciones Arqueológicas X, 119-138. Museo Arqueológico Provincial de Alicante, Alicante.

Martínez, I., Vilaplana, E., Such, I., Juan, J., García, M. A., 2014. Cabezo Pardo. Análisis instrumental de materiales de construcción de barro del yacimiento argárico. In: López Padilla, J. A. (Coord.), Cabezo Pardo (San Isidro/Granja de Rocamora), Excavaciones arqueológicas en el yacimiento de la Edad del Bronce, 330-378. MARQ, Alicante.

Melis, M. G., Albero, D., 2017. Archaeometric Analysis of Wall Coatings from The Chalcolithic Site of Su Coddu (Sardinia, Italy). Mediterranean Archaeology and Archaeometry 17(3), 191-200.

Miret i Mestre, J., 1992. Bòbila Madurell 1987-88. Estudi dels tovots i les argiles endurides pel foc. Arraona II, 67-72.

Moreno Onorato, A., 1993. El Malagón. Un asentamiento de la Edad del Cobre en el Altiplano de Cúllar-Chirivel. Tesis doctoral, Universidad de Granada.

Moreno-Alcaide, M., Compaña-Prieto J.M., 2018. Roman plasters and mortars from ancient Cosa (Tuscany-Italy). Mineralogical characterisation and construction from domus 10.1 (House with Cryptoporticus). Journal of Archaeological Science: Reports 19, 127-137.

Ortí, F., 1974. El Keuper del Levante español. Estudios Geológicos 30, 87-151.

Pastor Quiles, M., 2016. El estudio de los materiales constructivos de tierra del Cabezo del Polovar (Villena, Alicante): Aportación a las formas constructivas de dos pequeñas edificaciones campesinas de la Edad del Bronce en el Levante peninsular. DAMA. Documentos de Arqueología y Patrimonio Histórico I, 25-39.

Pastor Quiles, M., 2017. La construcción con tierra en arqueología. Teoría, método, técnicas y aplicación. Publicaciones Universidad de Alicante, Alicante. 
Pastor Quiles, M., 2019. La construcción con tierra en la Prehistoria reciente del Levante meridional de la península ibérica: materiales, técnicas y procesos constructivos. Tesis doctoral, Universidad de Alicante.

Pellicer Catalán, M., 1995. Las culturas del neolítico-calcolítico en Andalucía Oriental. Espacio, Tiempo y Forma, Serie I, Prehistoria y Arqueología 8, 81-134.

Pérez-López, A., 1991. El Trías de facies germánicas del sector central de la cordillera Bética. Tesis doctoral, Universidad de Granada.

Pérez-Valera, F., 2005. Estratigrafía y tectónica del Triásico en el sector oriental de la Cordillera Bética. Tesis Doctoral, Departamento Estratigrafía y Paleontología, Universidad de Granada.

Rivera Groennou, J. M., 2009. Micromorfología e interpretación arqueológica: aportes desde el estudio de los restos constructivos de un yacimiento argárico en el Alto Guadalquivir, Peñalosa (Baños de la Encina, Jaén). Cuadernos de Prehistoria y Arqueología de la Universidad de Granada 19, 339-360.

Rizo, C. E., García, J., Luján, A., 2000. Arqueología industrial en Villena: Contribución al estudio de las canteras de yeso del término municipal de Villena. Fundación José María Soler García, Villena.

Rosser, P., Fuentes, C., 2007. Tossal de les Basses: Seis mil años de historia de Alicante. Ayuntamiento de Alicante, Alicante.

Rubio de Miguel, I., 1985. En torno a la problemática del hábitat al aire libre en el neolítico peninsular. Cuadernos de Prehistoria y Arqueología de la Universidad Autónoma de Madrid 12, 153-161.

Sánchez Romero, M., 2015. Las arquitecturas de lo cotidiano en la Prehistoria reciente del sur de la península Ibérica. In: Díez Jorge, M.E. (Ed.), Arquitectura y mujeres en la historia: 19-58. Síntesis, Madrid.

Sandalinas, C., Ruiz-Moreno, S., López-Gil, A., Miralles, J., 2006. Experimental confirmation by Raman spectroscopy of a $\mathrm{Pb}-\mathrm{Sn}$-Sb triple oxide yellow pigment in sixteenth-century Italian pottery. Journal of Raman Spectroscopy 37(10), 1146-1153.
Schiffer, M., 1983. Toward the Identification of Formation Processes. American Antiquity 48(4), 675-706.

Schüle, W., Pellicer, M., 1966. El Cerro de la Virgen, Orce (Granada). Excavaciones Arqueológicas en España 46. Ministerio de Cultura, Madrid.

Scrivener, K. L., Taylor, H. F. W., 1993. Delayed ettringite formation: a microstructural and microanalytical study. Advances in Cement Research 5(20), 139-146

Sendova, M., Zhelyaskov, V., Scalera M., Ramsey, M., 2005. Micro-Raman spectroscopic study of pottery fragments from the Lapatsa tomb, Cyprus, ca 2500 BC. Journal of Raman Spectroscopy 36(8), 829-833.

Shaffer, G., 1993. An archaeomagnetic study of wattle and daub building collapse. Journal of Field Archaeology 20, 59-75.

Striova, J., Lofrumento, C., Zoppi, A., Castellucci, E.M., 2006. Prehistoric Anasazi ceramics studied by micro-Raman spectroscopy. Journal of Raman Spectroscopy 37(10), 1139-1145.

Surendran, S.M., Ravi, R., Subramani G. S., Chattopadhyay, S., 2017. Characterization of Ancient Mortars of Veppathur Temple. International Journal of Civil Engineering and Technology 8(4), 2132-2139.

Tuinstra, F., Koenig, J. L., 1970. Raman spectrum of graphite. The Journal of Chemical Physics 53(3), 1126-1130

Vilaplana, E., Martínez, I., Such, I., Juan, J. 2011. Presencia de carbonato cálcico $\left(\mathrm{CaCO}_{3}\right)$ recarbonatado en un probable fragmento constructivo de la ocupación neolítica cardial de Benàmer. In: Torregrosa Giménez, P., Jover Maestre, F. J., López Seguí, E. (Dirs.), Benàmer (Muro d’Alcoi, Alicante) Mesolíticos y neolíticos en las tierras meridionales valencianas, Serie de Trabajos Varios SIP, 112, Diputación de Valencia, Valencia.

Whitney, D.N, Evans, B.W., 2010. Abbreviations for names of rock-forming minerals. American Mineralogist 95, 185-187.

Zuo, J., Xu, C., Wang, C., Yushi, Z., 1999. Identification of the pigment in painted pottery from the Xishan site by Raman microscopy. Journal of Raman Spectroscopy 30(12), 1053-1055. 